\title{
El legado islámico en los cuidados psiquiátricos. El Maristán de Granada
}

\section{A herança islâmica em atendimento psiquiátrico. $\mathrm{O}$ Maristan de Granada Islamic heritage in psychiatric cares. The Maristan of Granada}

\author{
Alfonso Fernández Vázquez ${ }^{1}$, Rubén Mañá Ares² \\ ${ }^{1}$ Graduado Universitario en Enfermería por la Universidad de Salamanca. \\ ${ }^{2}$ Graduado Universitario en Historia por la Universidad de Santiago de Compostela \\ Cómo citar este artículo en edición digital: Fernández Vázquez, A. y Mañá Ares, R. (2016). El legado islámico en los cuidados \\ psiquiátricos. El Maristán de Granada. Cultura de los Cuidados (Edición digital), 20(45). Disponible en: < http://dx.doi. \\ org/10.14198/cuid.2016.45.06> \\ Correspondencia: Rúa París $21 \mathrm{H} 4^{\circ} \mathrm{D}$. 15707. Santiago de Compostela (A Coruña) \\ Correo electrónico: fonsinhox@gmail.com \\ Recibido: 8/12//2015; Aceptado: 15/05/2016
}

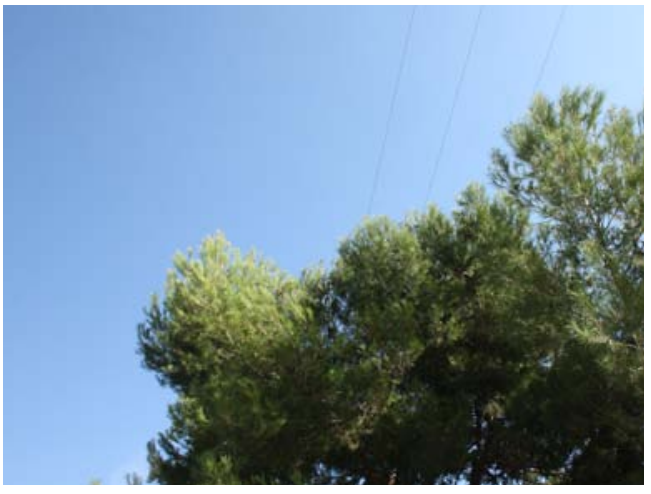

ABSTRACT

During the Middle Ages, Muslims influenced significantly in the health sciences, initiating a process of clumping of existing knowledge and the inclusion of new contributions. The aim of this article is to contextualize the conception of madness and care realised in medieval Islamic culture and to analize the structure and organization of the Maristan of Granada, main health sanitary institution of al-Ándalus and founded in 1367 by the emir Muhammad V. In order to get as much relevant data available to provide details of the enriched Arab-Islamic society of the Middle Ages vision, various historical documents and other literature sources they were examined. The findings of this review show the importance of Bimaristan of Granada as a promoter of health quality care to psychiatric patients in the Iberian Peninsula, including dietary-based therapies, hydrotherapy, massage, bloodletting and drug prescriptions. Decent and honorable treatment of the psychiatric patients would be imitated by some Christian psychiatric institutions in the peninsula since the 15 th century.

Keywords: conception of madness, medieval Islamic culture, Maristan of Granada, health quality care, psychiatric patient.

\section{RESUMO}

Durante a Idade Média, os mulçumanos influenciaram significativamente as ciências da saúde, iniciando um processo de aglutinação de conhecimento existente e a inclusão de novas contribuições. O objetivo do presente estudo é contextualizar o conceito de loucura e cuidado com base na cultura islâmica medieval e recordar a estrutura organizacional do Maristan de Granada, principal instituição de saúde de al-Andalus, fundada em 1367 pelo emir Muhammad V. Para obter os dados relevantes disponíveis, a fim de fornecer detalhes 
da sociedade árabe-islâmica, enriquecidos da visão medieval, foram consultados diversos documentos históricos e outras fontes bibliográficas. As conclusões desta avaliação destacam a importância da Bimaristan de Granada, como um promotor de cuidados de saúde com qualidade dirigidos aos doentes mentais na Península Ibérica, incluindo terapias dietéticas, hidroterapia, massagem, sangria e prescrição medicamentosa. Ademais, tratamentos dignos e respeitosos aos doentes mentais seriam imitados em algumas instituições psiquiátricas cristãs na Península, desde o século XV.

Palavras chave: concepção de loucura, a cultura islâmica medieval, Maristan de Granada, cuidados cuidados de qualidade, paciente psiquiátrico

\section{RESUMEN}

Durante la Edad Media los musulmanes influyeron significativamente en las ciencias de la salud, iniciando un proceso de aglutinamiento del conocimiento existente y la inclusión de nuevas aportaciones. El objetivo del presente artículo es contextualizar la concepción de la locura y los cuidados realizados en la cultura islámica medieval y rememorar la estructura y organización del Maristán de Granada, institución sanitaria principal de al-Ándalus y fundada en 1367 por el emir Muhammad V. Con el propósito de obtener la mayor cantidad de datos relevantes disponibles para proporcionar una visión enriquecida en detalles de la sociedad araboislámica de la Edad Media, se examinaron diversos documentos históricos y otras fuentes bibliográficas. Los hallazgos obtenidos de este examen resaltan la importancia del bimaristán de Granada como promotor del cuidado asistencial de calidad al paciente psiquiátrico en la Península Ibérica, incluyendo terapias basadas en la dietética, la hidroterapia, los masajes, las sangrías y la prescripción farmacológica. El trato digno y honroso al enfermo mental sería imitado por algunas instituciones psiquiátricas cristianas en la península a partir del siglo XV.

Palabras clave: concepción de la locura, cultura islámica medieval, Maristán de Granada, cuidado asistencial calidad, paciente psiquiátrico.

\section{INTRODUCCIÓN}

Los cuidados de psiquiátricos constituyen uno de los principales ámbitos de enfermería, cuyas técnicas se fundamentan en evidencias científicas y cada vez se alejan más de la metafísica y las tradiciones. A lo largo de la historia esta disciplina ha experimentado numerosas transiciones y cambios hasta lograr la situación actual. Durante la Edad Media los cristianos, musulmanes y judíos, mayoritariamente, convivieron en el territorio ibérico. La confluencia de estas tres culturas en la Península Ibérica de forma simultánea y las constantes interacciones entre ellas enriquecieron el arte de las ciencias de la salud heredado de la Edad Antigua.

La cultura musulmana implantó grandes contribuciones a la atención del paciente psiquiátrico en la península procedentes de los prósperos núcleos intelectuales de Oriente. La fundación de bimaristanes fue una de las mejoras introducidas por la civilización islámica, precediendo al hospital moderno. La primera de estas instituciones en la Península Ibérica fue el Maristán de Granada, fundado en 1367 por Muhammad V, y que constituyó uno de los principales centros de atención a pacientes psiquiátricos de gran relevancia para musulmanes y cristianos en el territorio ibérico. A 
lo largo de este artículo se describirá la concepción de la locura de la sociedad islámica en la Edad Media y su actitud hacia los enfermos mentales, la fundación e importancia del Maristán de Granada, su organización y funcionamiento, y la repercusión histórica de esta institución a la atención del enfermo mental.

\section{EL ESPLENDOR DE LA MEDICINA EN EL MUNDO ISLÁMICO MEDIEVAL}

El período histórico que comprendió el apogeo del mundo islámico fue de enorme grandeza dentro del desarrollo de diferentes ciencias. La medicina fue uno de los ámbitos donde se consiguieron grandes progresos, perfeccionando el saber disponible en aquella época. En la cultura islámica medieval, la enfermedad era entendida como un estado esencialmente transitorio que desembocaba en la curación o la muerte (Dolls, 1987). A pesar del determinismo divino defendido por el Islam bajo la premisa "El médico trata, Dios cura" asumido por médicos y enfermos, los musulmanes se han sentido atraídos hacia el abordaje de las patologías, incluidas las enfermedades mentales (Franco-Sánchez, 1999).

En el mundo islámico se consiguió sintetizar el pensamiento greco-occidental con el oriental, abriendo una nueva corriente que resaltó durante el vigoroso período de expansión y conquista de estos pueblos. Tal fue su importancia que los diferentes escritos de sus autores más representativos ${ }^{1}$ consiguieron sintetizar todo el saber hasta el momento, consiguiendo que sus textos fuesen utilizados en las facultades de medicina europeas hasta el siglo XVII, y aún son empleados actualmente en países como India y Pakistán (Bárcena, 2001).
Este valor de compilación que se le atribuye a la medicina árabe comienza en tiempos del Imperio de Bizancio cuando cristianos nestorianos son expulsados de él y se asientan en Persia en el 489 durante el Imperio Sasánida. Estos se encargaron de fundar una escuela de medicina en la ciudad de Gondishapur llegando a convertirse, al poco tiempo, en los grandes mediadores entre la cultura griega y oriental con la traducción de importantes obras médicas de la antigüedad. Con la conquista de Persia por los árabes en el siglo VIII, todo este capital inmaterial fue asimilado en un principio y posteriormente extendido, siendo Bagdad el nuevo centro cultural por excelencia donde prosiguió la traducción de obras clásicas de enorme valor en el campo de la medicina y filosofía. Este trabajo con fuentes, principalmente griegas, fue consecuencia en gran medida a que los primeros musulmanes siguieron las palabras de su profeta Mahoma, que determinaba lo siguiente: "Buscad el saber aunque hayáis de ir a China", "Quien deje su casa para dedicarse a la ciencia, sigue los pasos de Alá" (Chediak, 2007, p.165). En obediencia a este mandato, los musulmanes enviaron expediciones por toda Siria, Bizancio, Armenia $\mathrm{y}$ otras partes en búsqueda de manuscritos ${ }^{2}$ (Said-Farah, 2008). El avance del saber acompañado de los éxitos militares permitió el extraordinario desarrollo de la cultura islámica. Acudiendo de nuevo a Mahoma, este afirmaba que "solo hay dos ciencias; la teología (salvación del alma) y la medicina (salvación del cuerpo)", destacando la importancia de la medicina (Chediak, 2007, p. 166). Esta primera asimilación de los diferentes saberes médicos estuvo protagonizada por eruditos que en muchos

\footnotetext{
${ }^{1}$ Pongamos por caso, a autores como Avicena, Rhases, Averroes, Al-Mayusi o Albucasis.

${ }^{2}$ Los árabes tenían especial interés en los manuscritos de los clásicos griegos tales como Antíoco de Atenas, Apolonio, Aristóteles, Arquímedes, Dioscórides, Doroteo de Sidón, Euclides, Galeno, Herón, Hipócrates, Platón, Polemón, Tolomeo, Sócrates o Tales.
} 
casos no eran musulmanes. De este modo, tenemos constancia de cristianos nestorianos, judíos o zoroastrianos; esto es evidente cuando empezamos a estudiar los primeros hospitales en el mundo islámico debido a que los médicos eran elegidos en virtud de sus saberes, no de su fe (Bárcena, 2001).

No obstante, el desempeño de árabes y persas islamizados no se reducía a la mera traducción y transmisión del saber médico antiguo. Progresivamente, el mundo islámico recogería el testigo de los griegos en la transmisión y difusión del pensamiento intelectual, sistematizando de forma lógica el conocimiento antiguo, desarrollándolo e impregnándolo con su identidad propia.

Las ciencias tomadas por el islam viajaron a España desde Oriente entre los siglos VIII y IX. Bajo el emirato de Córdoba en el siglo X, la España árabe comienza su labor médica propia, dando inicio a la verdadera Edad de Oro de las ciencias en al-Ándalus en los siglos X y $\mathrm{XI}$, siendo su máximo exponente cultural la ciudad de Córdoba, denominada "La Bagdad de Oriente", el único centro civilizado de Europa en aquella época (García del Real, 1944).

\section{CONCEPCIÓN DE LA LOCURA EN LA SOCIEDAD ISLÁMICA MEDIEVAL}

La sociedad musulmana general entendió la locura como la posesión de espíritus o criaturas mágicas como genios (djinn o jinn), seres inteligentes y de grandes saberes, y que dominan los actos de aquellos inocentes a los que poseen. La palara que más se emplea para designar al demente es majnun, que significa literalmente "poseído por un jinn". La asociación locura y posesión está en la base de multitud de creencias y prácticas relacionadas con la religión y la magia y era aceptada universalmente por la sociedad islámica del medie- vo (Dols, 1987; Rodríguez, 2002). La creencia popular de la locura se hermana con la poesía o el amor, como revela el argumento de la célebre historia de "Layla y Majnun" del S.VII, presente en la literatura árabe, turca y persa hasta nuestros días. Este relato distinguido narra la historia de Qays y Layla, dos jóvenes intensamente enamorados, pero cuyo amor no permite el padre de Layla, comprometiendo en matrimonio a la joven con otro hombre. Qays huye al desierto semidesnudo ante la imposibilidad de tener a Layla, bajo la denominación de majnun (loco) atribuida por sus vecinos por su obsesión por la joven. Allí compone poemas para ella a la que sólo volverá a ver una vez más a lo largo de su vida. El amor y la pasión unidos a la locura es una temática frecuente en la literatura árabe, hasta el punto de asociar el amor como una enfermedad mental.

En el ámbito de la medicina, la construcción de la noción de la locura de los médicos islámicos medievales estuvo saturada de matices de la teoría hipocrática humoral y las teorías de Galeno (Pérez, Baldessarini, Undurraga y Sánchez-Moreno, 2012). Ar-Razí, Avicena o Averroes, entre otros, afirman que las enfermedades mentales poseen un carácter somático, dependientes de cuatro humores - sangre, flema, bilis amarilla y bilis negra - y que, a su vez, poseen cuatro cualidades - calor, frío, sequedad y humedad -. Esta asimilación de los antiguos conceptos médicos emana del vasto proceso de traducción de la cultura clásica de especial importancia (Dols, 1992). Varios médicos medievales islámicos como Avicena, que vivió durante un tiempo en Granada, describieron determinados trastornos neuropsiquiátricos que podrían beneficiarse de un tratamiento (Carnevali y Masillo, 2007). La noción científica de la locura y el carácter transitorio de la enfermedad permitió el abordaje tera- 
péutico de los dementes con el fin de reducir su afectación.

A pesar de las distintas concepciones de la etiología de la locura, los enfermos mentales siempre fueron respetados intensamente en el Islam. El loco no debía ser excluido de la sociedad, siendo una garantía del fluir humano de una comunidad religiosamente ordenada (González Duro, 1994). La tradición islámica reconoce la necesidad de atención institucional a los dementes pobres, como afirman las palabras de Mahoma:

"No confiar a los ineptos los bienes que Dios os ha confiado, pero encargaos vosotros mismos de ellos, alimentarlos y vestirlos y hablarles siempre con un lenguaje dulce y honesto" (Corán IV, versículo 4)

En el mundo islámico no existe el principio de caridad que favoreció a los dementes en la sociedad cristiana medieval de la Península Ibérica (Pileño, Morillo, Salvadores y Nogales, 2003). No obstante, el principio religioso musulmán Ihsan promovía la responsabilidad social de responder al bien con el bien, relacionado directamente con la bondad hacia los demás. Por este motivo, se realizaban acciones a favor de los desfavorecidos, lo que incluye a los enfermos mentales, que iban desde darles cobijo y manutención hasta la fundación de instituciones específicas para su atención por las clases sociales más altas.

Las familias con patrimonio suficiente para la atención de un miembro demente, lo recluían en una habitación, pero mostrando un trato honroso al enfermo y satisfaciendo todas sus necesidades. No obstante, aquellos locos inofensivos sin filiación ni recursos vagaban por las ciudades engrosando el grupo de los indigentes o mendicantes. El demente sólo era encerrado en caso de alterar el orden público habilitando, en un primer momento, celdas en las cárceles y, posteriormente, habitaciones o salas de los bimaristanes u hospitales (FrancoSánchez, 1999).

\section{LOS MARISTANES ISLÁMICOS}

Los bimaristanes islámicos eran centros asistenciales para enfermos mentales y físicos e instituciones educativas para la difusión de las profesiones sanitarias. La denominación de bimaristanes (o maristanes, forma popular de abreviar) está sujeta a diferentes interpretaciones por parte de diversos autores en relación con su origen. Autores como Bárcena (2001) sostienen que esta palabra proviene del persa y que responde al hospital (bimar-, enfermo) y (-istán, lugar, casa o asilo), debido a que la lengua árabe carecía de una terminología científica precisa. Otros autores, como Torres Balbás (1944) o Dols (1992), además de asignarle el nombre de hospital, atribuyen el término a la época de la romanización de la Península Ibérica argumentando que son una continuidad en el mundo islámico de los albergues llamados xenodocheion que se encontraban en ambos lados de los caminos transitados por viajeros. Estas instituciones solían ser actos de caridad individual de monarcas o personajes de importancia de la época islámica. Hacia el final de la Edad Media, en la mayoría de los maristanes no quedaron más que enfermeros mentales, derivando el significado de este término en una acepción similar al de manicomio (Torres, 1944). El conocimiento y las enseñanzas de los científicos islámicos implicaron que las instituciones predecesoras de los hospitales modernos en el mundo conocido - el nosocómeion bizantino, el maristán islámico y el hospital europeo - difirieran entre sí, tanto en lo relacionado con la atención a los pacientes, como a sus funciones, metodología y continuidad del conocimiento adquirido (Said-Farah, 2008). 
En relación a la fundación del primer bimaristán hay diferentes posiciones. Autores como Torres (1944) o Schipperges (1973) sostienen que el primer maristán o manicomio se constituyó bajo el mandato del califa Omeya al-Walid ibn 'Abd al-Malik en la ciudad de Bagdad en el 714, secundado por varias fuentes que afirman de su existencia hacia el año 765 (González Duro, 1994). Las mil y una noches, célebre obra literaria que recopila cuentos tradicionales orientales, afirma la existencia de un maristán para dementes en Bagdad en torno a las fechas que sostienen los investigadores anteriormente mencionados y que podría referirse a esa institución.

"Y contestó el visir: “'Oh mi señor! ¿qué te parecería una visita al asilo de los locos, al maristán, que tantas veces quisimos ver juntos, sin haber ido todavía? Porque opino que los locos son personas dotadas de un entendimiento diferente al nuestro, y que hallan entre las cosas relaciones que los que no están locos no distinguen nunca, y que son visitados por el espíritu. ¿Y acaso esa visita levante la tristeza que pesa sobre tu alma y dilate tu pecho!" Y contestó el sultán: “Por Alah, ¡oh visir mío! vamos a visitar a los locos del maristán!'”' (Anónimo, 2008)

No obstante, la mayoría de los eruditos sostienen que fue en tiempos del califa Harum ar-Rashid (786-809) cuando se creó el primer maristán en la ciudad de Bagdad, hacia el año 800, bajo la dirección del médico Yibril Ibn Bajtishu (Díaz, 1993), justificados en las evidencias históricas comprobadas que se poseen actualmente. También, prácticamente existe consenso en situar la brillante academia médica y hospital de la ciudad persa de Gondishapur como su antecedente, que presentó gran importancia para los primeros médicos y traductores islámicos (Rashed, 2005). En lo que no hay debate es en el aumento del número de bimaristanes en Bagdad durante los siglos IX y X. Cien años más tarde aparecieron estos hospitales en diferentes ciudades dando paso a la eclosión que se produce en el siglo XII en relación a estas construcciones en Próximo Oriente.

En El Cairo se funda uno de los principales maristanes de la época hacia el año 872 por orden de Ahmad Ibn Tulún, primero de los cinco tuluníes pertenecientes a la primera dinastía independiente del Egipto islámico (868905). En el siglo XII, el maristán de Damasco, construido durante el reinado de Nuruddín Ibn Imaduddín Zenguí (1118-1174), sultán de Siria y Egipto, era uno de los más grandes de la época (Gorini, Baggieri, y di Giacomo, 2004). Cuando Saladino conquistó Egipto en 1171, fundó en El Cairo otro nuevo hospital, descrito y muy elogiado por el célebre viajero valenciano lbn Yubayr con motivo de su visita a la actual capital egipcia (Torres, 1944). Finalmente, se funda en 1284 en El Cairo el hospital al-Mansurí, por orden del sultán mameluco Saifuddín Qala'ûn al-Alfí (gobernante entre 1279-1290) que podía albelgar a ocho mil pacientes de ambos sexos. Hacia finales del siglo XIII existen registros de la fundación de bimaristanes en Damasco, Bagdad, Antioquía, Alepo, Jerusalén y El Cairo, confirmando la expansión de las instituciones sanitarias en el oriente musulmán (Issa, 1928).

La introducción del bimaristán en el occidente musulmán fue tardía, con la fundación de dos instituciones en Marruecos y Fez, y con mayor posterioridad aún en el territorio peninsular. En este último caso, también podemos comprobar cómo hay hipótesis dispares en relación con el primer bimaristán peninsular. A pesar de todo, los primeros datos seguros de los que existe constancia corresponden al caso granadino. 


\section{EL MARISTÁN DE GRANADA}

En este proceso de fundaciones de bimaristanes, y pareciendo imitar al hospital anteriormente fundado en Marruecos por Abu Yusuf ibn Ya'qub, surge el granadino, construido por el emir Muhammad V entre 1365 y 1367 a lo largo de veinte meses. La escasez de datos sobre instituciones sanitarias en al-Ándalus contrasta con la abundancia de hospitales y monasterios del norte de la península, al igual que el mundo islámico oriental (Franco-Sanchez, 1999). A la hora de acudir a fuentes que nos permitan obtener información sobre este edificio, además de cronistas y viajeros de la época o posteriores ${ }^{3}$, es de vital importancia el texto fundacional de éste, el cual se conserva en una lápida de mármol en forma de arco de herradura. Este hospital, además de su importancia a nivel médico, está enmarcado dentro de la importante labor política realizada por el gobierno de Muhammad V.

"Loor a Dios. Ordenó la construcción de este maristán, como muestra de amplia misericordia para con los más débiles enfermos musulmanes y como acercamiento útil [...]. Y tuvo lugar el comienzo de su construcción en la decena de mediados del mes de Muharram del año setecientos setenta y siete (27 de septiembre- 8 de octubre de 1365), y se terminó lo que se propuso, para lo que asigno legados píos, en la docena de mediados de sawwal del año setecientos sesenta y ocho (9-18 de junio de 1367). Dios no descui- da la recompensa de los que realizan buenas obras ni frustra el esfuerzo de los benefactores. Salve Dios a nuestro Muhammad, sello de los profetas, a su familia y a sus compañeros, todos (Acién, 1995)."

El maristán se ubicó en un amplio solar cercano al río Darro, al inicio de la ladera que asciende a la Alcazaba, ubicado enfrente al Baño del Nogal y de la Puerta de los Tableros (más conocido como puente del Cadí). Historiadores indican que el Muhammad V debió de aprovechar la estructura de varios edificios previos que confluían en un edificio de planta única, hipótesis que justifica la escasa duración de las obras. Según la descripción de Ibn al-Jatíb, citada en los excelentes trabajos de FrancoSánchez (1999), el resultado final resultó ser un edificio rectangular que albergaba en su interior un patio central ornamentado con plantas, árboles frutales y flores cuyas dimensiones ascienden a $38.3 \mathrm{~m}$ de largo y $26.5 \mathrm{~m}$ de ancho, con una alberca ${ }^{4}$ tapial de aproximadamente $14 \mathrm{~m}$ de largo y $5 \mathrm{~m}$ de ancho, rodeado por dependencias en todo su derredor similar a las casas nazaríes de la época (García Granados y Salvatierra, 1984, 1990; Girón, 1978). La alberca conectaba con dos inmensos leones ${ }^{5}$ situados en el centro de los lados largos de la alberca que surtían por sus bocas agua a través de canales al depósito nazarí (García Granados, Girón y Salvatierra, 1989). Las dependencias eran cuadradas, íntegramente amuebladas, de aproximadamente $2.50 \mathrm{~m}$ cada lado y comuni-

\footnotetext{
${ }^{4}$ La alberca es una construcción árabe que podía efectuarse excavando en la tierra directamente o mediante el uso de materiales de construcción, generando un espacio en el que almacenar agua similar a un estanque artificial. Su finalidad puede ser ornamental, para el riego o los conocidos albercones, que recogían el agua de la lluvia o canales cercanos para su posterior uso y almacenaje. Actualmente, podemos encontrar albercas en distintos restos arqueológicos en la ciudad nazarí de Granada como, por ejemplo, en la Alhambra de Granada, o en la Alcazaba de la ciudad de Málaga.

${ }^{5}$ Esta descripción recuerda la Fuente de los leones conservada en la Alhambra de Granada. No obstante, los hallazgos arqueológicos y registros escritos afirman que los dos leones del Maristán de Granada eran de mayor tamaño, sentados sobre los cuartos traseros y los detalles fueron esculpidos con mayor minuciosidad y precisión.

${ }^{6}$ Autores como Torres (1944) afirman que ambos leones fueron trasladados del Maristán de Granada, y actualmente suministran el agua de la alberca de la Torre de las Damas en la Alhambra de Granada.
} 


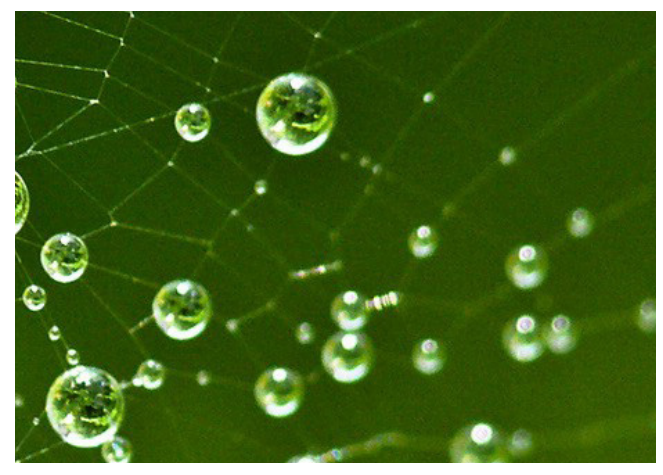

cadas entre sí a través de una galería de $80 \mathrm{~cm}$ de ancho que permitía recorrer las dependencias sin necesidad de acceder al patio interior. Las habitaciones se encontraban abiertas hacia el interior por puertas de aproximadamente $1 \mathrm{~m}$ de ancho y cerradas hacia el exterior del edificio (Girón, 1986). En cada una de las esquinas del interior del edificio se situaba una escalera que permitía el paso a la planta superior de la institución. La planta superior contenía dependencias de mayor superficie, siendo un total de 26 dependencias las que componen el edificio. La heterogeneidad de los elementos decorativos de materiales como yeso, madera, lana, lino, seda u oro del maristán embellecían el interior del edificio, emplazando a sus residentes en un entorno idílico. Los muros del edificio se encontraban libres de elementos decorativos en su exterior, a excepción de la parte central en la que se situaba la puerta de entrada (Francho-Sánchez, 1999).

Teniendo en cuenta que la beneficencia daba muestra de la calidad del gobernante virtuoso, el monarca Muhammad V procuró contribuir a reforzar su imagen de autoridad político-religiosa mediante el bimaristán nazarí, sin escatimar en ostentaciones y riquezas en su construcción. Esto lo hizo a través del waf que era un subsidio enfocado a proteger enfermos pobres y débiles - incluyendo los dementes - de forma que recibían una cobertu- ra básica que pudiese atender sus necesidades (Franco-Sánchez, 1999). La capacidad de crear este auxilio se encontraba entre los grupos privilegiados de la sociedad, de forma más concreta entre las autoridades políticas.

\section{ORGANIZACIÓN Y FUNCIONAMIENTO DEL MARISTÁN}

A pesar de que en la lápida fundacional indica que se atendían a pobres enfermos musulmanes, el Maristán de Granada es considerado por la historiografía clásica habitualmente como un centro que alojó mayoritariamente a enfermos mentales, debido, principalmente, a la escasez de referencias en las fuentes de médicos que ejercieran en sus dependencias, argumento insuficiente debido a la parquedad de referencias en otras instituciones sanitarias en las que sí parece ser que se atendían toda patología existente (Peláez, 2011). Además, el viajero alemán Jerónimo Munzer, menciona una casa de locos en su escrito al rey don Fernando tras visitar la ciudad nazarí, apoyando la idea de que esta institución se dedicó al cuidado de enfermos mentales. Este debate está fuertemente enmarcado dentro de la búsqueda del primer hospital para dementes en la Península, pero las fuentes no nos permiten determinar esta categoría al hospital nazarí aunque fuese esa función la que tuviera más peso (Dols, 1992). Relacionado con este debate, podemos citar el Hospital d'Innocentes, Folles i Orats de Valencia como el primer psiquiátrico del mundo. Con todo, la polémica no está zanjada debido a que el hospital londinense de Bethlem también reclama esta distinción (Fernández-Vázquez, 2015).

El tratamiento del alienado en el Islam medieval se aleja del internamiento indefinido del individuo recluyéndolo en un centro ajeno al ámbito social, como indica Garriga (2010), 
juicio fundamentado en la cognición médica del carácter transitorio de las enfermedades. En la sociedad islámica medieval, el enfermo mental tenía acceso a distintas opciones de tratamiento: (a) el tratamiento médico o admisión en un maristán; (b) recibir una bendición tras el peregrinaje a la tumba de un individuo religioso de renombre; (c) asesoramiento personalizado y legal, o (d) consultar a un curandero para obtener ungüentos, pócimas o talismanes. La elección dependía de los síntomas, disponibilidad de los médicos, creencias personales, la influencia de familiares o amigos, o la presencia de curanderos cercanos (Pérez et al., 2012). La escasez de registros del desempeño realizado en el maristán granadino y la similitud de la organización y praxis en otros maristanes citados en fuentes disponibles, nos hacen presuponer que la organización y el abordaje terapéutico realizado en el Maristán de Granada es similar al de otras bimaristanes islámicos de la época.

Los maristanes se organizaban en torno a una administración, un dispensario, una farmacia donde se preparaban las recetas médicas, almacenes, una mezquita $y$, con frecuencia, una biblioteca especializada. En la cúspide jerárquica del desempeño profesional de los maristanes se encontraba el administrador elegido por la autoridad política de la ciudad y, frecuentemente, ajeno al ámbito de las ciencias de la salud ${ }^{7}$. El director (qayyim) era el encargado de supervisar el funcionamiento del sanatorio, regular las funciones del personal sanitario y no sanitario de la institución y en algunos casos, la preparación y dispensación de pociones. Los médicos no residían en los bimaristanes, más bien, acudían periódicamente para evaluar la situación de los enfermos e instaurar, modificar o suprimir el tratamiento, refrendado en la descripción de Ibn Yubayr, traducido por Maíllo (1988) de la organización del maristán situado en Bagdad.

"Los médicos lo visitan cada lunes y jueves $y$ allí examinan el estado de los enfermos y prescriben el tratamiento que les es necesario. A su disposición están unos asistentes que se dedican a preparar los remedios y las comidas. Es un gran palacio con salas, cuartos y todas las comodidades de las viviendas reales. El agua entra allí desde el Tigris."

Los registros y descripciones existentes indican que en los bimaristanes existían asistentes del personal médico con funciones específicas, como indica la descripción de Ibn Yubayr, siendo muy probable que este colectivo desempeñara tareas propias de enfermería.

Dejando a un lado argumentos referidos a epidemias de peste o necesidades de guerra como motivadoras de la construcción del hospital, la institución parece tener albergado un modelo basado en la relación personal maestrodiscípulo en la cual este último recibía educación teórico-práctica donde trabajaba con enfermos, diagnosticando enfermedades y preinscribiendo dietas diarias y medicación (Arvide, 1983).

\section{TRATAMIENTOS ÁRABES EN LOS EN- FERMOS MENTALES}

La medicina árabe sostiene la curación en tres pilares: la dieta, los medicamentos y la cirugía. La dietética para los médicos árabes es la base del tratamiento o incluso la única prescripción si la enfermedad no pide mayores intervenciones, como lo refleja Averroes:

"La prevención de la salud depende de dos cosas: el cuidado de la buena digestión y el cui-

\footnotetext{
${ }^{7}$ Entre las excepciones se encuentra el médico Abu Ishaq Ibrahim al-Dani, médico que ostentó el cargo de administrador en el maristán de Marruecos (Castells, 1992).
} 
dado de la evacuación de los excedentes del cuerpo. El primer objetivo se logra seleccionando los alimentos adecuados para el organismo tanto atendiendo a su calidad como a su cantidad y, también, al momento conveniente de su ingestión y distribución. El segundo, la evacuación de los excedentes, ha de cuidarse como el primero que se efectuará de dos maneras: mediante el ejercicio o mediante la ingestión de los medicamentos que expelen los excedentes del cuerpo" (Vázquez, 2001).

Con respecto a las enfermedades mentales, la locura se asienta en el individuo en presencia del desequilibrio entre humores de las teorías hipocráticas y galenas. Según los médicos árabes, la harmonía de los líquidos hipocráticos es influida por la ingesta alimenticia, destacando la relevancia de la nutrición y dietética en el paciente. Otras variables influyentes en los humores son el aire, la excreción, la actividad, el reposo, la actividad sexual, el sueño y las emociones. Digno de mención es indicar que Florence Nightingale, enfermera del siglo XIX y uno de los máximos exponentes de esta disciplina, señala enfatizó la importancia del entorno en la salud y describió algunos elementos que inciden en ella, como la ventilación, la temperatura, la iluminación, la dieta, la higiene o el ruido, coincidiendo en varios puntos de anclaje con la concepción islámica del medievo para la restitución de la salud (Nightingale, 1969).

El médico trata de modificar estos elementos con el fin de lograr la homeostasis interna del paciente y en consecuencia, la curación del alienado. La diversidad terapéu- tica es amplia, comprendiendo la dietética, la hidroterapia, los masajes, las sangrías y la prescripción farmacológica (Bárcena, 2001). Algunos facultativos árabes nombran ciertas terapias para enfermos mentales (Nogales, $2001)^{8}$. El viajero judío Benjamín de Tudela, testimonio recogido en la obra de Asher (1840), reportó lo siguiente con respecto a las terapéuticas árabes:

"Los pacientes dementes de toda la región son ingresados en este hospital. Por lo general son confinados y encerrados con la necesidad de recibir tratamientos específicos, [...] música para aliviar la melancolía, o baños para calmar maníaco-agitación [...]. Algunos se mantienen en las restricciones hasta que se recuperen un estado mental adecuado. Por lo menos una vez al mes, los supervisores hacen evaluaciones para determinar qué pacientes se han recuperado lo suficiente como para ser enviado de vuelta a sus casas"

Además de atención sanitaria, el bimaristán contó con cocinas y espacios de cultivo de productos medicinales dando lugar a un servicio farmacológico que interconectaba la medicina y la farmacología mediante la botánica. De estos terrenos conservamos interesantes documentos, los cuales también podían tener un objetivo ornamental y ambiental, aspecto enormemente valorado para el cuidado del cuerpo.

"Todo ha de contribuir a la curación de los enfermos. Se construye y se decora con la intención de actuar sobre el psiquismo de los internos, ya que se parte de la idea de que la belleza, basada en el equilibrio, la proporción y el ritmo, es sanadora" (Bárcena, 2001).

\footnotetext{
${ }^{8}$ Maimónides recomendaba para combatir la melancolía el empleo de la música y de conversaciones para los enfermos melancólicos. Rhazes (850-923) confiaba en la sangría para tratar el frenesí, la apoplejía y la migraña. Abul-Casim (S.X) ordenaba practicar la cauterización en casos de apoplejía y de epilepsia.

${ }^{9}$ Texto traducido de la obra The Itinerary of Rabbi Benjamin de Tudela de Asher realizada en 1840.
} 


\section{REPERCUSIÓN DEL MARISTÁN DE GRA- NADA}

Tras la conquista de Granada por los Reyes Católicos, éstos destinaron el Maristán de Granada a la Casa de la Moneda, edificio que continuó en poder de la Corona hasta la primera mitad del siglo XVII. A finales del siglo XVIII, esta institución se utilizó para usos industriales, debido a su estado de abandono (Torres, 1944). Ante esta situación de ruina y precariedad, el arquitecto don Baltasar Romero pidió licencia para su derribo, no sin el descontento del pueblo español demoliendo el inmueble a finales de agosto de 1843.

Esta institución influyó en el establecimiento y organización de los sanatorios posteriores de la Península Ibérica. Gimeno (1908) informó del trato honorable y digno al enfermo mental de la sociedad islámica, siendo tomada esta actitud por los cristianos en la construcción y organización de asilos para dementes en Europa a partir del siglo XV. Según afirma Fernández-Vázquez (2015), el padre Jofré se inspiró en la atención y cuidados psiquiátricos desempeñados en el Maristán de Granada para la fundación del primer hospital psiquiátrico del mundo occidental, el Hospital de los Inocentes de Valencia.

\section{BIBLIOGRAFÍA}

- Acién, M. (1995). Inscripción conmemorativa de la construcción de un Maristán. En J. Bermúdez (Eds.), Arte islámico en Granada. Propuesta para un Museo de la Alhambra (pp.340-342). Granada: Patronato de la Alhambra-Comares.

- Anónimo (2008). Las mil y una noches. Madrid: Alianza Editorial.

- Arvide, L. M. (1983). Nota sobre la práctica y la enseñanza de la medicina en la España musulmana de los siglos XIXII. El Ms árabe 887 de El Escorial. Dynamis, 3, 313-339.

- Asher, A. (1840). The Itinerary of Rabbi Benjamin de Tudela. London. Disponible en http://wdb.ugr. es/ proyecto_viajeros/recursos/textos/judios/tudela_ asher_1840.pdf

- Bárcena, C. G. (2001). El Bimaristán, un modelo de hos- pital islámico: Historia de los primeros centros psiquiátricos del mundo. Revista Natural Medicatrix, 62, 6-11.

- Carnevali, R. y Masillo, A. (2007). Brief history of psychiatry in the Islamic world. Journal of the International Society for the History of Islamic Medicine, 6, 97-101.

- Castells, M. (1992). Médecins andalous au Maghreb. En Historia, ciencia y sociedad: actas del II Coloquio Hispano-Marroquí de Ciencias Históricas (Granada, 6-10 noviembre 1989) (pp. 123-124). Madrid: Agencia Española de Cooperación Internacional.

- Chediak, E. (2007). Tres médicos árabes: Rhases, Averroes, Avicena. Bogotá: Academia Nacional de Medicina. p 165.

- Díaz, F. (1993). La vida cotidiana en la España musulmana. Madrid: Edaf.

- Dols, M. W. (1987). Insanity and its treatment in Islamic society. Medical History, 31, 1-14.

- Dols, M. W. (1992). Majnun. The Madman in Medieval Islamic Society. Oxford: Clarendon Press.

- Fernández-Vázquez, A. (2015). Cambio en la noción de la locura. El Hospital de los Inocentes de Valencia. Cultura de los cuidados, 19(41), 106-113. doi:http://dx.doi. org/10.14198/cuid.2015.41.13.

- Franco-Sánchez, F. (1999). La asistencia al enfermo en al-Andalus: los hospitales hispanomusulmanes. En C. Álvarez (Eds), La medicina en Al-Andalus (pp. 135-173). Granada: Fundación El Legado Andalusí, Conserjería de Cultura, Junta de Andalucía.

- García del Real, E. (1944). La medicina árabe española. Revista nacional de educación, 45, 16-33.

- García Granados, A., Girón, F., y Salvatierra, V. (1989). El Maristán de Granada, un hospital islámico. Granada: Asociación Española de Neuropsiquiatría.

- García Granados, A., y Salvatierra, V. (1984). El Maristán de Granada: entre la fantasía romántica y la realidad arqueológica. Revista de Arqueología, 42, 16-25.

- García Granados, A., y Salvatierra, V. (1990). Un edificio rehabilitado en el siglo XIV: el Maristán de Granada. Córdoba: Diputación Provincial.

- Garriga, D. (2010). Del bimaristán al hospital psiquiátrico: historia de la enfermería y la salud mental en el Islam. Madrid: Asociación Nacional de Enfermería de Salud Mental.

- Gimeno, J. (1908). La Casa de los Locos y el Hospital de Nuestra Señora de Gracia. Apuntes históricos (1425-18081908). Zaragoza: Imp. La Editorial.

- Girón, F. (1978). En torno al Maristán (hospital árabe) de Granada. Asclepio, 30-31, 223-231.

- Girón, F. (1986). Los hospitales en la España musulmana. Jano, 30(711), 66-79.

- González Duro, E. (1994). Historia de la Locura en España. Tomo I. Siglos XIII-XVII. Madrid: Temas de hoy.

- Gorini, R., Baggieri, G., y di Giacomo, M. (2004). 


\section{Cultura de las Cuidados}

Internement et Traitement de la Maladie Mentale au Moyen-Âge islamiste: l'exemple des Bîmarîstâns au Maroc et en Syrie. Antropo, 7, 99-104.

- Issa, A. (1928). Histoire des Bimaristans (hopitaux): a lépoque islamique. El Cairo: Paul Barbey.

- Maíllo, F. (1988). A través del Oriente. El siglo XII ante los ojos, Rihla. Barcelona: Ediciones del Serbal.

- Nightingale, F. (1969). Notes on Nursing. What it is and what it is not. New York: Dover Publications, Inc.

- Peláez, A. (2011). El Maristán de Granada al servicio del poder nazarí: el uso político de la caridad. En A. M. Carballeira (Eds.), Estudios onomástico-biográficos de alAndalus XVI (pp. 131-170). Madrid: Consejo Superior de Investigaciones Científicas.

- Pérez, J., Baldessarini, R., Undurraga, J., y SánchezMoreno, J. (2012). Origins of Psychiatric Hospitalization in Medieval Spain. Psychiatric Quarterly, 83(4), 419-430. doi:10.1007/s11126-012-9212-8

- Pileño, M. E., Morillo, F. J., Salvadores, P., y Nogales, A. (2003). El enfermo mental. Historia y cuidados desde la época medieval. Cultura de los cuidados, 13, 29-35.

- Rashed, R. (2005). Encyclopedia of the history os Arabic Science: Technology, alchemy and life sciences. Vol 3. London: Routledge.

- Rodríguez, F. (2002). Sobre la Locura en las Sociedades Musulmanas Tradicionales. Frenia, 2, 147-160.

- Said-Farah, M. (2008). El Maristán y Al-madrasa: Hospital-Escuela de medicina. Archivos de la Sociedad Española de Oftalmología, 83(3), 201-204.

- Schipperges, H. (1973). La medicina en el medievo árabe. En P. Lain (Eds.), Historia Universal de la Medicina, vol. 3 (pp.105-109). Barcelona: Ed. Salvat.

- Torres, L. (1944). El Maristán de Granada, Al-Andalus, 9, 481-498.

- Vázquez, M. C. (2001). La medicina andalusí: La medicina del Tabib y su proyección social. En F. Roldán, y I. Hervás (Eds.), El saber en al-Ándalus: textos $y$ estudios, volumen III (pp. 46-54). Sevilla: Universidad de Sevilla.

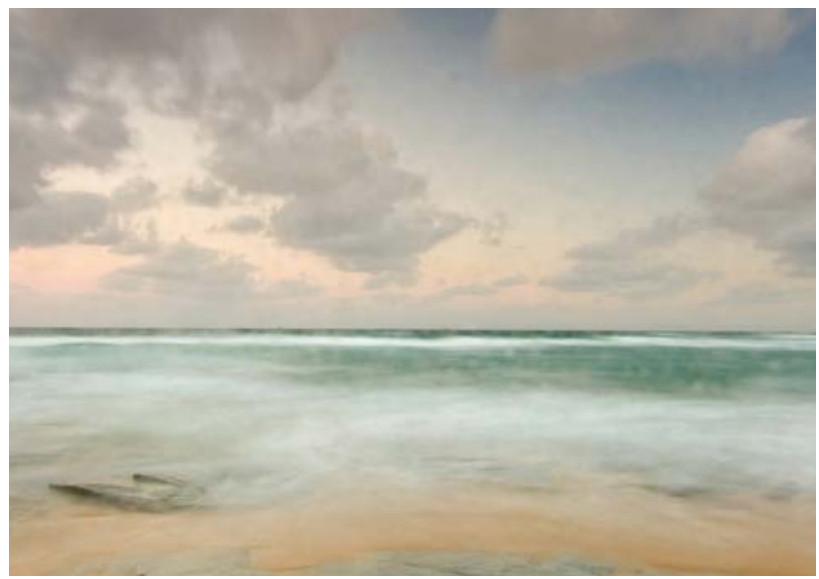

